\title{
Improved perioperative outcomes and reduced inflammatory stress response in malignant robotic- assisted colorectal resections: a retrospective cohort study of 298 patients
}

Pedja Cuk ( $\sim$ pedja.cuk@rsyd.dk)

Sygehus Sønderjylland: Sygehus Sonderjylland https://orcid.org/0000-0003-0582-604X

\section{Randi Maria Simonsen}

Sygehus Sønderjylland: Sygehus Sonderjylland

Mirjana Komljen

Sygehus Sonderjylland

Michael Festersen Nielsen

Sygehus Sonderjylland

\section{Per Helligs $\varnothing$}

Sonderjylland Hospital: Sygehus Sonderjylland

\section{Andreas Kristian Pedersen}

Sygehus Sønderjylland: Sygehus Sonderjylland

\section{Christian Backer Mogensen}

Sygehus Sønderjylland: Sygehus Sonderjylland

\section{Mark Bremholm Ellebæk}

Odense University Hospital: Odense Universitetshospital

\section{Research}

Keywords: Colorectal cancer, robotic-assisted colorectal surgery, laparoscopic colorectal surgery

Posted Date: March 5th, 2021

DOl: https://doi.org/10.21203/rs.3.rs-278055/v1

License: (c) (i) This work is licensed under a Creative Commons Attribution 4.0 International License. Read Full License 


\section{Abstract \\ Background}

Robotic-assisted surgery is increasingly implemented for the resection of colorectal cancer, although the scientific evidence for adopting this technique is still limited. This study's main objective was to compare short-term complications, oncological outcomes, and the inflammatory stress response after colorectal resection for cancer performed laparoscopic or robotic-assisted.

\section{Methods}

We conducted a retrospective cohort study comparing the robotic-assisted approach to laparoscopic surgery for elective malignant colorectal neoplasm. Certified colorectal and da Vinci $®$ robotic surgeons performed resections at a Danish tertiary colorectal high volume centre from May 2017 - March 2019. We analyzed the two surgical groups using uni- and multivariate regression analyses to detect differences in intra- and postoperative clinical outcomes and the inflammatory stress response.

\section{Results}

Two hundred and ninety-eight patients were enrolled in the study. Significant differences favoring roboticassisted surgery was demonstrated for; length of hospital stay (4 days, interquartile range (4-5) versus 5 days, interquartile range (4-7), $\mathrm{p}<0.001$ ) and intraoperative blood loss $(50 \mathrm{~mL}$, interquartile range (20$100)$ versus $100 \mathrm{~mL}$, interquartile range (50-150), $\mathrm{p}<0.001)$ compared to laparoscopic surgery. The inflammatory stress response was significantly higher after laparoscopic compared to robotic-assisted surgery reflected by an increase in C-reactive protein concentration (exponentiated coefficient $=1.20,95 \%$ confidence interval (1.04-1.40), $p<0.001)$. No differences between the two groups were found concerning mortality, microradical resection rate, conversion to open surgery and surgical or medical short-term complications.

\section{Conclusion}

Robotic-assisted surgery is feasible and can be safely implemented for colorectal resections. The roboticassisted approach, when compared to laparoscopic surgery, was associated with improved intra- and postoperative outcomes. Extensive prospective studies are needed to determine the short and long-term outcomes of robotic surgery for colorectal cancer.

\section{Introduction}

Colorectal cancer is a common malignant disease with a global annual incidence of 1.8 million (2017) and an incidence rate of 23.2/100,000 inhabitants (1). The treatment approach is primarily surgical, and 
if possible, a minimally invasive approach should be preferred. Laparoscopic colorectal surgery (LCS) has existed for more than 20 years. The many benefits of LCS compared to open surgery include less postoperative pain, fewer wound complications and improved cosmetic outcomes (2). The disadvantages of LCS include a prolonged learning curve, suboptimal ergonomics, and suboptimal visual exposure of the surgical field due, in part, to tremor caused by the assistant (3-5). The oncological results of LCS and open colorectal surgery are identical, and the three to five-year survival and recurrence rates of the two surgical methods are comparable (2).

Within the last 15 years, robotic-assisted colorectal surgery (RCS) has increasingly been used to treat colorectal cancer. Weber et al. was the first to describe this surgical method in 2002 (6). RCS is associated with longer operating times and higher total costs when compared to laparoscopic surgery. However, robotic-assisted surgery has additional benefits, including a reduced risk of conversion to open surgery, improved postoperative morbidity, reduced intraoperative bleeding, comparable oncologic rates, and a faster establishment of bowel function (7-11). Furthermore, robotic-assisted surgery offers a stable and better visual exposure and instruments with a higher degree of flexibility to enhance surgical dissection quality $(7,12)$. It can be challenging to determine the differences between intra- and postoperative outcomes as both surgical methods are minimally invasive. The incidence of lower morbidity rates in RCS $(13,14)$ compared to LCS surgery may be associated with a lower inflammatory stress response due to less tissue trauma and better hemostasis (15). Therefore, we hypothesized that RCS induces a lower surgical trauma with improved surgical morbidity than LCS.

This study's main objective was to compare short-term complications, oncological outcomes, and inflammatory stress response after resection for cancer performed with robotic-assisted or laparoscopic colorectal surgery.

\section{Methods}

\section{Study design}

The study was conducted according to The Strengthening the Reporting of Observational Studies in Epidemiology (STROBE guidelines) (16).

It was performed as a retrospective cohort study at the Surgical Department, Hospital of Southern Jutland, Denmark - a tertiary care hospital performing robotic and laparoscopic-assisted colorectal surgery.

We minimized bias by only including procedures performed by certified surgeons in colorectal cancer and robotic-assisted surgery (da Vinci®, Intuitive Surgical, Inc., Sunnyvale, CA, USA). Data collection was obtained by review of medical records from May 2017 - March 2019. Only patients undergoing intended curative elective colorectal resections were included in the study. Established search criteria were endoscopic and histopathological confirmed adenocarcinoma of the colon (cecum, right colon, 
transverse colon, left colon, sigmoid colon) and rectum. Patients undergoing a palliative or emergency resection were excluded, as were patients with benign lesions.

\section{Outcome measurements and data collection}

Data were registered in an electronic database, Research Electronic Data Capture (REDCap $\left.{ }^{\circledR}\right)$, hosted by the Open Patient data Explorative Network (OPEN), Odense University Hospital and Department of Clinical Research (17). We collected information regarding demographics (age, gender, body mass index (BMI), and the American Society of Anesthesiologists (ASA) physical status classification system. Intra- and postoperative data included the following parameters: conversion to open surgery, estimated intraoperative blood loss, operative time, length of hospital stay, time to first flatus and stool, surgical and medical complications. Postoperative surgical and medical complications occurring within 90 days postoperatively were defined as severe if graded $\geq 3$ points according to Clavien Dindo classification (18). Anastomotic leakage was graded in severity according to Rahbari from A (conservative treatment), B (active re-intervention without laparoscopy or laparotomy), and C (re-intervention with laparoscopy or laparotomy) (19). The leakage between intestinal walls of anastomosis ends were detected by either 1) pneumoperitoneum on computer tomography (CT) or 2) dehiscence between intestinal ends identified by either re-laparoscopy, re-laparotomy or endoscopy. Pathological data included the TNM classification of malignant tumors (TNM), number of harvested lymph nodes, rate of microradical resection and preoperative neoadjuvant chemotherapy or combined chemo-/radiotherapy. To evaluate the inflammatory stress response, CRP and leukocyte counts were registered from the first postoperative day until discharge.

\section{Surgical procedure}

Patients were allocated to either RCS or LCS depending on the first available scheduled surgical time. Before surgery, patients underwent a multidisciplinary cancer conference. All patients received preoperative antibiotic prophylaxis (Piperacillin/Tazobactam $3+0,5 \mathrm{~g}$ and Metronidazole 1,5 g), antithrombotic (Dalteparin $5000 \mathrm{IE}$ ), compression stockings, urinary catheter and a nasogastric tube. Surgical procedures were performed either laparoscopically or totally robotic-assisted. A da Vinci® robot, Xi model (Intuitive Surgical, Inc., Sunnyvale, CA, USA), was used in the RCS procedures. Two-dimensional or threedimensional (3D) laparoscopy was used in LCS procedures depending on the surgeon's choice. Complete mesocolic excision (CME) was not applied, but a minimum D2 resection was performed in all colonic resections. Low or abdominoperineal anterior resections were based on total or partial mesorectal excision principles. The following trocars were applied in RCS procedures: a $12 \mathrm{~mm}$ umbilical trocar for camera guidance, three $8 \mathrm{~mm}$ trocars for surgical instruments, and one $8 \mathrm{~mm}$ trocar for the surgical assistant. Traditionally in LCS four or five 5- and 12-mm trocars were applied. A transverse muscle splitting incision was performed for right-sided tumors, the specimen was extracted, and an extracorporeal isoperistaltic single-layer, end-to-end, handsewn anastomosis was performed. A horizontal incision in the left iliacal fossa was used for specimens extraction in left-sided and rectal tumours, and an end-to-end stapled anastomosis was performed. In low anterior resection procedures, a protective loop 
ileostomy was performed, dependent on patients' comorbidity or in case of a low colorectal anastomosis $(<5 \mathrm{~cm})$.

\section{Data analysis}

Descriptive statistics were performed for each variable. Categorical variables were presented with frequencies and percentage and compared using Fisher's exact or Pearson chi-square test depending on Cochrane's rule (20). Non-categorical variables were presented with median and interquartile range and compared using the Wilcoxon rank-sum test. Logistic regression was used to examine the complications between the two surgical techniques. Depending on the outcome's distribution, poisson regression or a negative binomial regression was used to examine how the different surgical techniques influenced the length of hospital stay, time to first flatus, stool and harvested lymph nodes. Log transformation with a mixed effect model estimated CRP and leukocyte differences between the two operation methods across all days. All generalized linear models were adjusted for T-stage, ASA-score and neoadjuvant chemotherapy. Models followed the one in ten rule for fixed effects and the one in twenty rule for random effects. The models' fit was completed using quantile-quantile plots of the deviance residuals and residuals for each time point for the generalized linear model and mixed effect models, respectively. A pvalue of less than 0.05 was seen as statistically significant, and no correction for multiple testing was utilized.

\section{Results}

Three-hundred and sixty-one patients operated for colorectal cancer were identified, and of these, 298 patients (RCS, $n=143$ (38\%); LCS, $n=155$ (52\%) fulfilled the inclusion criteria (Fig.

1). All baseline characteristics were similar across the two groups except for neoadjuvant chemotherapy (RCS group (7.0\%) versus LCS group (16.8\%), $p=0.010$ and combined neoadjuvant chemo-/radiotherapy (RCS group (4.2\%) versus LCS group (13.5\%), $p=0.005)$, (Table 1).

\section{Intraoperative outcomes}

There was a statistically significant difference in the distribution of colonic and rectal resections between the two surgical methods (RCS group (77.6\%) versus LCS group (57.4\%), $p=0.015$ ) (Table 2). Median intraoperative blood loss was $50 \mathrm{~mL}(20-100)$ in the RCS group versus $100 \mathrm{~mL}(50-150) \mathrm{mL}$ in the LCS group, $\mathrm{p}<0.001$, (Table 2).

\section{Postoperative outcomes and complications}

The time of hospitalization was reduced by a median of one day in the RCS group compared to the LCS group ( 4 days $(4-5)$ versus 5 days (4-7), p < 0.001). Time to first stool favoured LCS (2 days $(1-3)$ versus RCS, 3 days $(2-3), p=0.033)$. No differences in postoperative surgical and medical complications were demonstrated between the two groups (Table 3). CRP concentration was significantly lower in the RCS group on day 1, 3 and 4 postoperatively. Similarly, the leukocyte concentration was significantly 
lower in the RCS group on postoperative day 4 but otherwise did not differ significantly in the postoperative period (Fig. 2, Table 3).

\section{Pathologic outcome}

No differences were observed in $\mathrm{T}-\mathrm{N}$ stage or amount of harvested lymph nodes between the patients operated with RCS or LCS (Table 4). A significant number of patients with distant disease occurred in the $\operatorname{LCS}$ group $(n=3,(1.9 \%)$ compared to the RCS group $(n=0), p=0.043)$.

\section{Multivariate regression analysis}

A multivariate regression analysis was performed with adjustment for ASA-score, T-stage and neoadjuvant chemotherapy for surgical and medical complication rates, operative time, length of hospital stay, intraoperative blood loss, time to first flatus and stool, amount of harvested lymph nodes, CRP and leukocyte count (Table 5). The multivariate analysis demonstrated a statistically significant increase in surgical time $(\mathrm{IRR}=1.03,95 \% \mathrm{Cl}=1.01-1.04, \mathrm{p}<0.001)$ and a reduction in the amount of harvested lymph nodes $(\mathrm{IRR}=0.92,95 \% \mathrm{Cl}=0.88-0.95, \mathrm{p}<0.001)$ in the LCS group. The remaining intra- and postoperative outcomes outlined in the univariate analysis were not affected by the regression analysis.

\section{Discussion}

The present study demonstrates a significant reduction in length of hospital stay, intraoperative blood loss and inflammatory stress response measured by CRP in patients undergoing colorectal resection for cancer with RCS compared to the LCS. A multivariate regression analysis with adjustment for ASA-score, T-stage and neoadjuvant chemotherapy demonstrated an additional reduction in the operative time and the amount of harvested lymph nodes favoring RCS. No difference was found in surgical or medical morbidity, time to first flatus or stool, conversion rate to open surgery, postoperative leukocyte count or the microradical resection rate between the two groups in multivariate analyses.

Comparison of RCS and LCS for malignant disease remains poorly investigated. In 2012, a prospective randomized controlled trial examined the length of stay as a primary outcome and reported no difference between the two surgery techniques (2). The ROLARR study from 2017 demonstrated that the risk of conversion was higher for obese patients $\left(\mathrm{BMI}>30 \mathrm{~kg} / \mathrm{m}^{2}\right)$ undergoing surgery for rectal cancer (7).

Recent systematic reviews and meta-analyses comparing surgical efficacy and safety of RCS and LCS favor RCS concerning several intra- and postoperative outcomes. These outcomes include lower conversion rates, intraoperative blood loss, decreased overall morbidity, earlier hospital discharge, and earlier establishment of bowel function $(8,13,14,21-23)$. In this study, there was a non-significant conversion rate of $1.4 \%$ in the RCS group and $3.9 \%$ in the LCS group, $p=0.187$. Solaini et al. reported a significant conversion rate to open surgery in LCS resections (RR 1.7; 95\% $\mathrm{Cl}(1.1-2.6), \mathrm{p}=0.02$ ). This finding has also been confirmed in other systematic reviews and meta-analyses $(3,8,24-27)$. Despite more patients in our population receiving combined chemo-/radiotherapy, no difference in conversion 
rates could be detected between the surgical groups. However, conversion rates for patients receiving neoadjuvant radiotherapy were higher. Factors that may complicate the surgical procedure and induce this higher conversion rate include radiotherapy to the pelvic floor causing fibrosis, edema, inflammation and necrosis (28). Neoadjuvant radiotherapy is associated with a higher risk of postoperative surgical complications and delayed perineal wound healing following abdominoperineal resection (29). None of these complications were overrepresented in our study.

In contrast to other studies, operative time was no longer for RCS than LCS $(4,10,24-26,30)$. However, previous meta-analyses have been conducted on predominantly observational studies whereby there is a risk of misinterpretation of total procedure times and surgical times (31-33). The widely criticized fact by RCS is the setup and docking time of the robotic console. The prolonged surgical times cannot be attributed to simple factors but rather the joint effort of the limited number of certified RCS surgeons, dedicated operating nurses and/or anesthesiology team. Improved RCS technological development and a transition from DaVinci Si ${ }^{\circledR}$ to the Xi ${ }^{\circledR}$ model and a dedicated robotic team can reduce total operation time. Several studies have confirmed a reduction in both docking and total operating times using the DaVinci Xi ${ }^{\circledR}$ model, with an average of 21 cases needed to reach a statistically significant reduction in the docking time (34-36). The technological development of RCS and the da Vinci Xi ${ }^{\circledR}$ model's introduction allows the surgeon more freedom and the ability to perform even technically demanding procedures that previously have been difficult to perform laparoscopically. The stable and precise high definition camera, which the surgeon independently can maneuver and the higher degree of free movement of robotic arm joints contributes to better hemostasis (37). Although both surgical modalities are minimally invasive, RCS is presumed to be associated with a gentler manipulation with organs.

Postoperative CRP levels were significantly lower in RCS and can predict the inflammatory stress response induced by surgery. There is sparse literature reporting on the systemic inflammatory response in RCS compared to LCS (38). Previous studies have mostly compared the systemic inflammatory response in RCS to open colorectal surgery. RCS was associated with a lower inflammatory stress response compared to open surgery $(39,40)$. A prospective, non-randomized study comparing RCS ( $\mathrm{n}=$ $30)$ and LCS surgery $(n=120)$ for early gastric adenocarcinoma reported a lower postoperative CRP and interleukin-6 response in the LCS group. In this study, there was an unequal distribution of patients, lack of randomization and usage of older da Vinci robotic ${ }^{\circledR}$ technology. These factors may have contributed to a lower postoperative inflammatory response in the LCS group.

Regarding oncological outcomes, the microradical resection rate was not statistically different between groups. Our study showed a significantly lower amount of harvested lymph nodes in LCS group from the multivariate analysis. Many existing reports examine the rate of harvested lymph, and the majority report no differences between the two operation methods $(2,10,25-27,41,42)$. However, a large Danish register-based observational study including a total of 8104 LCS and 511 RCS procedures for colorectal cancer showed the risk of achieving a microradical resection in colon cancer was significantly higher using LCS, and higher but non-significant for rectal cancer in patients undergoing RCS (43). 
The most important limitation of this study is the retrospective design. Patients were preoperatively balanced between the two surgical procedure groups regarding age distribution, BMI and ASA-score. There was a selection of patients who had received neoadjuvant oncological treatment in favor of LCS. These patients did not have a significantly increased rate of conversion or postoperative morbidity in either of the surgical groups. To minimize the risk of selection bias, we performed a multivariate regression analysis adjusting for clinically relevant confounders. Apart from time to first stool, none of the univariate analyses were non-significant by these adjustments.

\section{Conclusion}

RCS is a well-established surgical method. This study demonstrated the superiority of RCS compared to LCS in regards to surgical safety and efficacy. Lower intraoperative blood loss, shortened hospital stay and a lower postoperative inflammatory stress response was observed in the RCS group. There is a need to evaluate the potential benefits of RCS, focusing on differences in inflammation caused by the two surgical methods in randomized and prospective studies.

\section{Abbreviations}

\section{LCS}

laparoscopic colorectal surgery

RCS

robotic-assisted colorectal surgery

$\mathrm{BMI}$

body mass index

ASA

American Society of Anesthesiologists

TNM

TNM classification of malignant tumors

CRP

C-reactive protein

CT

computer tomography

CME

complete mesocolic excision

$\mathrm{OR}$

odds ratio

$\mathrm{Cl}$

confidence interval

IRR

incidence rate ratio 
EC

exponentiated coefficient

\section{Declarations}

\section{Ethical approval and consent to participate}

The study was approved by the Danish Patient Safety Authority, case number N 0 3-3013-2926/1, and the Danish Data Protection Agency, file number 19/12742.

\section{Consent for publication}

Not applicable.

\section{Competing interests}

The authors declare no competing interests.

\section{Availability of data and materials}

Data can be required from the corresponding author.

\section{Funding}

The authors have no funding interests to declare.

\section{Acknowledgements}

We would like to thank Caroline Moos for critical review and proofreading of this manuscript.

\section{Author's contribution}

Study design: PC, RMS and MBE. Data analysis: PC and AKP. Manuscript preparation: PC. Manuscript review: MK, MFN, PH, CBM, MBE and PC. The manuscript was proved by the authors.

\section{References}

1. Collaborators GBDCC. The global, regional, and national burden of colorectal cancer and its attributable risk factors in 195 countries and territories, 1990-2017: a systematic analysis for the Global Burden of Disease Study 2017. Lancet Gastroenterol Hepatol. 2019;4(12):913-33 DOI: 10.1016/S2468-1253(19)30345-0.

2. Park JS, Choi GS, Park SY, Kim HJ, Ryuk JP. Randomized clinical trial of robot-assisted versus standard laparoscopic right colectomy. Br J Surg. 2012;99(9):1219-26 DOI: 10.1002/bjs.8841. 
3. Lee SH, Kim DH, Lim SW. Robotic versus laparoscopic intersphincteric resection for low rectal cancer: a systematic review and meta-analysis. Int J Colorectal Dis. 2018;33(12):1741-53 DOI: 10.1007/s00384-018-3145-0.

4. Holmer $C$, Kreis ME. Systematic review of robotic low anterior resection for rectal cancer. Surg Endosc. 2018;32(2):569-81 DOI: 10.1007/s00464-017-5978-y.

5. Trastulli S, Farinella E, Cirocchi R, Cavaliere D, Avenia N, Sciannameo F, et al. Robotic resection compared with laparoscopic rectal resection for cancer: systematic review and meta-analysis of short-term outcome. Colorectal Dis. 2012;14(4):e134-56 DOI: 10.1111/j.1463-1318.2011.02907.x.

6. Weber PA, Merola S, Wasielewski A, Ballantyne GH. Telerobotic-assisted laparoscopic right and sigmoid colectomies for benign disease. Dis Colon Rectum. 2002;45(12):1689-94; discussion 95-6 DOI: $10.1007 /$ s10350-004-7261-2.

7. Jayne D, Pigazzi A, Marshall H, Croft J, Corrigan N, Copeland J, et al. Effect of Robotic-Assisted vs Conventional Laparoscopic Surgery on Risk of Conversion to Open Laparotomy Among Patients Undergoing Resection for Rectal Cancer: The ROLARR Randomized Clinical Trial. JAMA. 2017;318(16):1569-80 DOI: 10.1001/jama.2017.7219.

8. Solaini L, Bazzocchi F, Cavaliere D, Avanzolini A, Cucchetti A, Ercolani G. Robotic versus laparoscopic right colectomy: an updated systematic review and meta-analysis. Surg Endosc. 2018;32(3):1104-10 DOI: $10.1007 /$ s00464-017-5980-4.

9. Ng KT, Tsia AKV, Chong VYL. Robotic Versus Conventional Laparoscopic Surgery for Colorectal Cancer: A Systematic Review and Meta-Analysis with Trial Sequential Analysis. World J Surg. 2019;43(4):1146-61 DOI: 10.1007/s00268-018-04896-7.

10. Kim CW, Kim CH, Baik SH. Outcomes of robotic-assisted colorectal surgery compared with laparoscopic and open surgery: a systematic review. J Gastrointest Surg. 2014;18(4):816-30 DOI: 10.1007/s11605-014-2469-5.

11. Duan BS, Zhao GH, Yang H, Wang Y. A Pooled Analysis of Robotic Versus Laparoscopic Surgery for Colon Cancer. Surg Laparosc Endosc Percutan Tech. 2016;26(6):523-30 DOI: 10.1097/SLE.0000000000000359.

12. Xu H, Li J, Sun Y, Li Z, Zhen Y, Wang B, et al. Robotic versus laparoscopic right colectomy: a metaanalysis. World J Surg Oncol. 2014;12:274 DOI: 10.1186/1477-7819-12-274.

13. Genova P, Pantuso G, Cipolla C, Latteri MA, Abdalla S, Paquet JC, et al. Laparoscopic versus robotic right colectomy with extra-corporeal or intra-corporeal anastomosis: a systematic review and metaanalysis. Langenbecks Arch Surg. 2020 DOI: 10.1007/s00423-020-01985-x.

14. Trastulli S, Cirocchi R, Desiderio J, Coratti A, Guarino S, Renzi C, et al. Robotic versus Laparoscopic Approach in Colonic Resections for Cancer and Benign Diseases: Systematic Review and MetaAnalysis. PLoS One. 2015;10(7):e0134062 DOl: 10.1371/journal.pone.0134062.

15. Roy S, Evans C. Overview of robotic colorectal surgery: Current and future practical developments. World J Gastrointest Surg. 2016;8(2):143-50 DOl: 10.4240/wjgs.v8.i2.143. 
16. von Elm E, Altman DG, Egger M, Pocock SJ, Gotzsche PC, Vandenbroucke JP, et al. The Strengthening the Reporting of Observational Studies in Epidemiology (STROBE) statement: guidelines for reporting observational studies. J Clin Epidemiol. 2008;61(4):344-9 DOI: 10.1016/j.jclinepi.2007.11.008.

17. Harris PA, Taylor R, Thielke R, Payne J, Gonzalez N, Conde JG. Research electronic data capture (REDCap)-a metadata-driven methodology and workflow process for providing translational research informatics support. J Biomed Inform. 2009;42(2):377-81 DOI: 10.1016/j.jbi.2008.08.010.

18. Dindo D, Demartines N, Clavien PA. Classification of surgical complications: a new proposal with evaluation in a cohort of 6336 patients and results of a survey. Ann Surg. 2004;240(2):205-13 DOI: 10.1097/01.sla.0000133083.54934.ae.

19. Rahbari NN, Weitz J, Hohenberger W, Heald RJ, Moran B, Ulrich A, et al. Definition and grading of anastomotic leakage following anterior resection of the rectum: a proposal by the International Study Group of Rectal Cancer. Surgery. 2010;147(3):339-51 DOI: 10.1016/j.surg.2009.10.012.

20. Kroonenberg PM, Verbeek A. The Tale of Cochran's Rule: My Contingency Table has so Many Expected Values Smaller than 5, What Am I to Do? The American Statistician. 2018;72(2):175-83 DOI: 10.1080/00031305.2017.1286260.

21. Ngu JC, Ng YY. Robotics confers an advantage in right hemicolectomy with intracorporeal anastomosis when matched against conventional laparoscopy. J Robot Surg. 2018;12(4):647-53 DOI: $10.1007 / \mathrm{s} 11701-018-0793-6$.

22. Waters PS, Cheung FP, Peacock O, Heriot AG, Warrier SK, O'Riordain DS, et al. Successful patientoriented surgical outcomes in robotic vs laparoscopic right hemicolectomy for cancer - a systematic review. Colorectal Dis. 2020;22(5):488-99 DOI: 10.1111/codi.14822.

23. Rausa E, Kelly ME, Asti E, Aiolfi A, Bonitta G, Bonavina L. Right hemicolectomy: a network metaanalysis comparing open, laparoscopic-assisted, total laparoscopic, and robotic approach. Surg Endosc. 2019;33(4):1020-32 DOI: 10.1007/s00464-018-6592-3.

24. Ma S, Chen Y, Chen Y, Guo T, Yang X, Lu Y, et al. Short-term outcomes of robotic-assisted right colectomy compared with laparoscopic surgery: A systematic review and meta-analysis. Asian J Surg. 2019;42(5):589-98 DOI: 10.1016/j.asjsur.2018.11.002.

25. Sun Y, Xu H, Li Z, Han J, Song W, Wang J, et al. Robotic versus laparoscopic low anterior resection for rectal cancer: a meta-analysis. World J Surg Oncol. 2016;14:61 DOI: 10.1186/s12957-016-0816-6.

26. Chang YS, Wang JX, Chang DW. A meta-analysis of robotic versus laparoscopic colectomy. J Surg Res. 2015;195(2):465-74 DOI: 10.1016/j.jss.2015.01.026.

27. Xiong B, Ma L, Huang W, Zhao Q, Cheng Y, Liu J. Robotic versus laparoscopic total mesorectal excision for rectal cancer: a meta-analysis of eight studies. J Gastrointest Surg. 2015;19(3):516-26 DOI: 10.1007/s11605-014-2697-8.

28. Evans J, Patel U, Brown G. Rectal cancer: primary staging and assessment after chemoradiotherapy. Semin Radiat Oncol. 2011;21(3):169-77 DOI: 10.1016/j.semradonc.2011.02.002.

29. Wasserberg N. Interval to surgery after neoadjuvant treatment for colorectal cancer. World $\mathrm{J}$ Gastroenterol. 2014;20(15):4256-62 DOI: 10.3748/wjg.v20.i15.4256. 
30. Casillas MA, Jr., Leichtle SW, Wahl WL, Lampman RM, Welch KB, Wellock T, et al. Improved perioperative and short-term outcomes of robotic versus conventional laparoscopic colorectal operations. Am J Surg. 2014;208(1):33-40 DOI: 10.1016/j.amjsurg.2013.08.028.

31. Kim JC, Lee JL, Yoon YS, Kim CW, Park IJ, Lim SB. Robotic left colectomy with complete mesocolectomy for splenic flexure and descending colon cancer, compared with a laparoscopic procedure. Int J Med Robot. 2018;14(5):e1918 DOI: 10.1002/rcs.1918.

32. Widmar M, Keskin M, Strombom P, Beltran P, Chow OS, Smith JJ, et al. Lymph node yield in right colectomy for cancer: a comparison of open, laparoscopic and robotic approaches. Colorectal Dis. 2017;19(10):888-94 DOI: 10.1111/codi.13786.

33. Lim DR, Min BS, Kim MS, Alasari S, Kim G, Hur H, et al. Robotic versus laparoscopic anterior resection of sigmoid colon cancer: comparative study of long-term oncologic outcomes. Surg Endosc. 2013;27(4):1379-85 DOI: 10.1007/s00464-012-2619-3.

34. van der Schans EM, Hiep MAJ, Consten ECJ, Broeders I. From Da Vinci Si to Da Vinci Xi: realistic times in draping and docking the robot. J Robot Surg. 2020;14(6):835-9 DOI: 10.1007/s11701-02001057-8.

35. Feng Z, Feng MP, Feng DP, Solorzano CC. Robotic-assisted adrenalectomy using da Vinci Xi vs. Si: are there differences? J Robot Surg. 2020;14(2):349-55 DOI: 10.1007/s11701-019-00995-2.

36. Morelli L, Guadagni S, Di Franco G, Palmeri M, Caprili G, D'Isidoro C, et al. Use of the new da Vinci $\mathrm{Xi}(\mathrm{R})$ during robotic rectal resection for cancer: a pilot matched-case comparison with the da Vinci Si(R). Int J Med Robot. 2017;13(1) DOI: 10.1002/rcs.1728.

37. Karcz WK, von Braun W. Minimally Invasive Surgery for the Treatment of Colorectal Cancer. Visc Med. 2016;32(3):192-8 DOI: 10.1159/000445815.

38. Park JY, Jo MJ, Nam BH, Kim Y, Eom BW, Yoon HM, et al. Surgical stress after robot-assisted distal gastrectomy and its economic implications. Br J Surg. 2012;99(11):1554-61 DOI: 10.1002/bjs.8887.

39. Zawadzki M, Krzystek-Korpacka M, Gamian A, Witkiewicz W. Comparison of inflammatory responses following robotic and open colorectal surgery: a prospective study. Int J Colorectal Dis. 2017;32(3):399-407 DOI: 10.1007/s00384-016-2697-0.

40. Krzystek-Korpacka M, Zawadzki M, Lewandowska P, Szufnarowski K, Bednarz-Misa I, Jacyna K, et al. Distinct Chemokine Dynamics in Early Postoperative Period after Open and Robotic Colorectal Surgery. J Clin Med. 2019;8(6) DOI: 10.3390/jcm8060879.

41. Memon S, Heriot AG, Murphy DG, Bressel M, Lynch AC. Robotic versus laparoscopic proctectomy for rectal cancer: a meta-analysis. Ann Surg Oncol. 2012;19(7):2095-101 DOI: 10.1245/s10434-0122270-1.

42. Lin S, Jiang HG, Chen ZH, Zhou SY, Liu XS, Yu JR. Meta-analysis of robotic and laparoscopic surgery for treatment of rectal cancer. World J Gastroenterol. 2011;17(47):5214-20 DOI: 10.3748/wjg.v17.i47.5214.

43. Fransgaard T, Pinar I, Thygesen LC, Gogenur I. Association between robot-assisted surgery and resection quality in patients with colorectal cancer. Surg Oncol. 2018;27(2):177-84 DOI: 
Figures

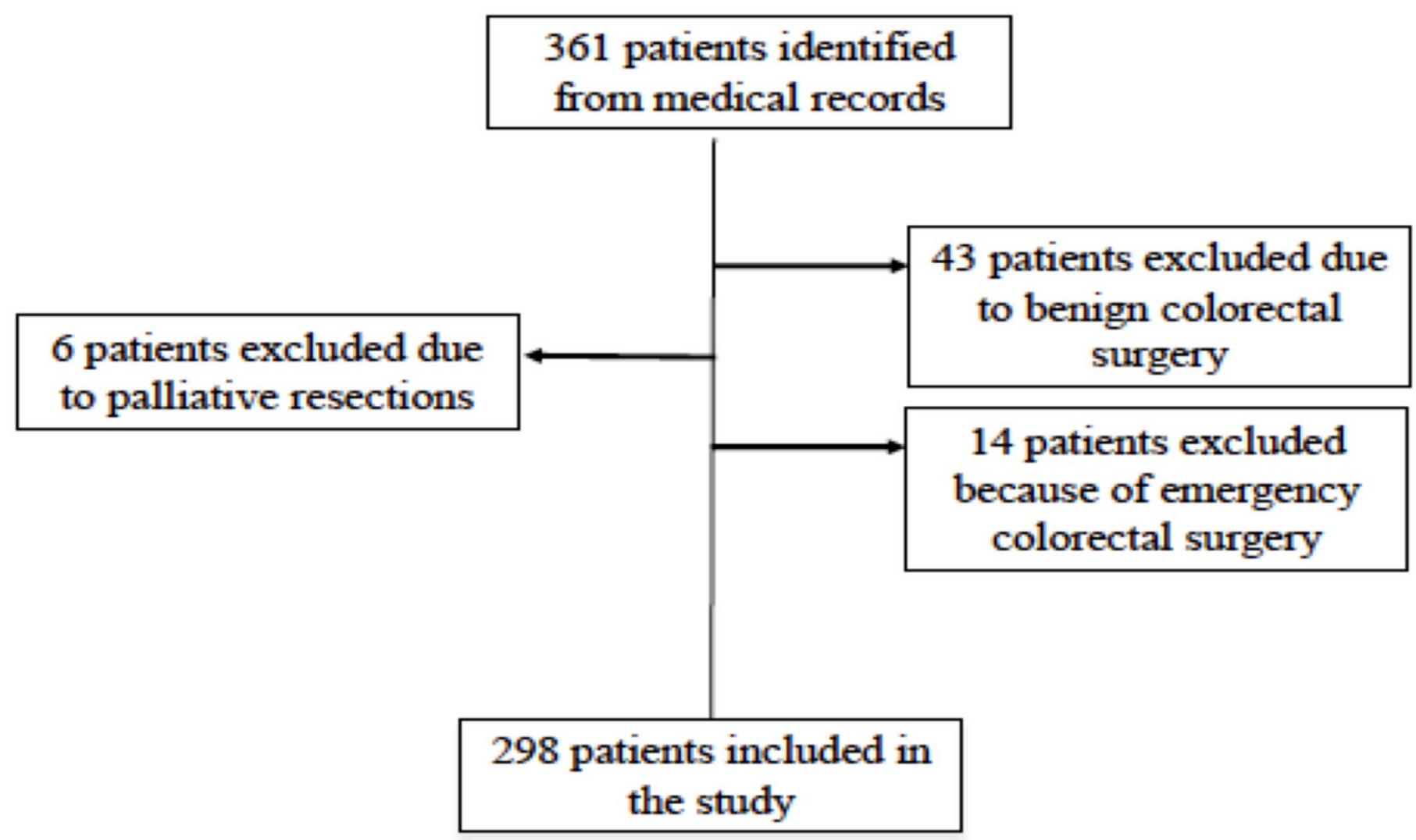

Figure 1

Flowchart of study population selection 

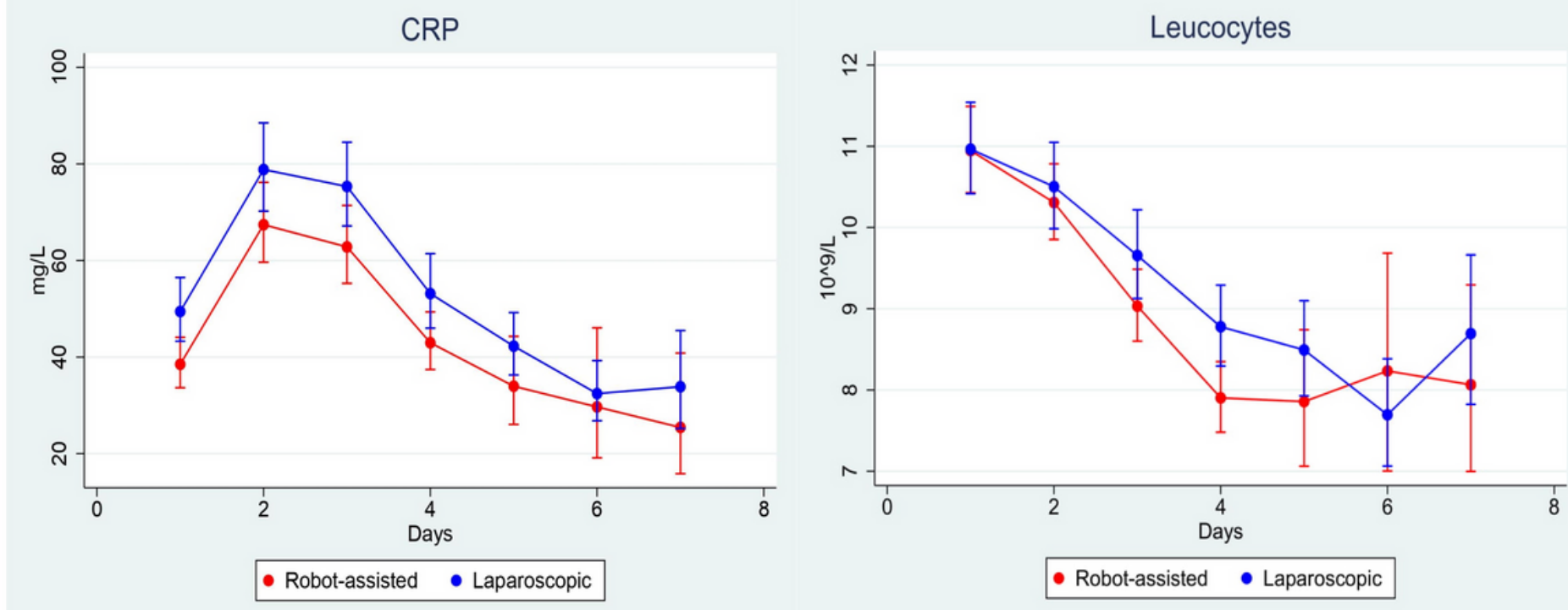

\section{Figure 2}

Distribution of postoperative C-reactive protein (CRP) level and leukocyte count day 1-7 in RCS versus LCS

\section{Supplementary Files}

This is a list of supplementary files associated with this preprint. Click to download.

- Table1.pdf

- Table2.pdf

- Table3.pdf

- Table4.pdf

- Table5.pdf 